\title{
Association of Free Fatty Acid (FFA), Fatty Acid Binding Protein (FABP) and Adiponectin with Tumor Necrosis Factor- $\alpha$ (TNF- $\alpha$ ) and Interleukin-6 (IL-6) Among Obese Non Diabetic Males
}

\author{
Yani Lina ${ }^{1,2}$, Gatot Susilo Lawrence ${ }^{3}$, Andi Wijaya ${ }^{1,2}$
}

$\mathrm{B}$ ACKGROUND: The prevalence of obesity has increased dramatically in recent years. It is commonly associated with type 2 diabetes, coronary artery disease, and hypertension. White adipose tissue (WAT) is a major site of energy storage and is important for energy homeostasis. WAT has been increasingly recognized as an important endocrine organ that secretes a number of biologically active "adipokines". The resultant higher FFA, FABP4, FABP5 concentration; and lower concentration of adiponectin is known to be correlated with inflammation. The aim of this study was to observe the correlation between FFA, FABP4, FABP5 and adiponectin with TNF- $\alpha$ and Interleukin-6 as markers of inflammation.

METHOD: The study was observational with a cross sectional design. The analysis was done on 69 male subjects aged 30-60 years with non diabetic abdominal obesity which is characterized by waist circumference (WC) $98.7 \pm 6.5 \mathrm{~cm}$ and fasting blood glucose $87.1 \pm$ $9.7 \mathrm{mg} / \mathrm{dL}$. FFA testing was performed by enzymatic colorimetric assay; whereas FABP4, FABP5, TNF- $\alpha$, adiponectin and IL-6 were performed by ELISA. All statistical calculations were performed with the SPSS 11.5 statistical software package. We used the Pearson or Spearman's rho correlation coefficient to assess the correlation between various anthropometric and biochemical measures. We also used path analysis Lisrel 8.30 for Windows.

RESULT: This study revealed that there was no correlation between FFA, FABP4 and adiponectin with TNF- $\alpha$ and Interleukin-6, whereas there was correlation between FABP5 with TNF- $\alpha$ and Interleukin-6. This study also showed there were correlations between WC and hsCRP $(r=0.314, p=0.000)$, WC and IL-6 $(r=$ $0.276, p=0.022)$, FFA and FABP4 $(r=0.263, p=0.029)$, FABP4 and WC $(r=0.249, p=0.039)$, FABP4 and BMI $(\mathrm{r}=0.311, \mathrm{p}=0.009)$, FABP5 and TNF- $\alpha(\mathrm{r}=0.408, \mathrm{p}=$ $0.000)$, FABP5 and FABP4 $(r=0.296, p=0.014)$, FABP5 and Interleukin- $6(\mathrm{r}=0.248, \mathrm{p}=0.04)$, Adiponectin and HDL-Cholesterol $(r=0.301, p=0.012)$.

CONCLUSION: Abdominal obesity might contribute to inflammation in obese nondiabetic males. This study indicated that in abdominal obesity, FFA may induce inflammation through FABP4 and FABP5. Advancing our understanding of the function and measurement of FABP4 and FABP5 serum concentration will give insight into the clinical diagnosis of obesity-related metabolic disorders.

KEYWORDS: Obesity, Waist Circumference, Free Fatty Acid (FFA), Fatty Acid Binding Protein (FABP), Adiponectin, TNF- $\alpha$, Interleukin-6, Inflammation.

\section{Introduction}

Obesity has become epidemic throughout the world and poses significant health and economic burdens to both developed and developing societies. Globally, the World Health Organization estimates that a billion adults have body mass index greater than $25 \mathrm{~kg} / \mathrm{m} 2$ and 300 million are obese with BMI over $30 \mathrm{~kg} / \mathrm{m} 2$. As obesity is usually associated with an increase in insulin resistance, it is not surprising that there has

${ }^{1}$ Prodia Clinical Laboratory, Jakarta

${ }^{2}$ Post Graduate Program in Clinical Biochemistry, Hasanuddin University, Makassar

${ }^{3}$ Medicine Department, Hasanuddin University, Makassar 
been an equally dramatic increase in the incidence of type 2 diabetes (T2DM). Although it is still being debated how accumulation of subcutaneous and/or visceral fat produces insulin resistance, which occurs predominantly in skeletal muscles, it has nevertheless become clear that elevated plasma levels of free fatty acids (FFA) are a major link between obesity and insulin resistance/T2DM. Plasma levels of FFA are commonly higher in obese than in non-obese individuals. The reason for this fact is that an increased number of fat cells, a decrease in FFA clearance and at a later stage, insulin resistance at the adipose tissue level result in increased lipolysis. The key initiating event is an increase in plasma FFA followed by increased uptake of FFA into muscle. This leads to intramyocellular accumulation of fatty acyl-CoA and diacylglycerol (DAG) and activation of protein kinase C/PKC (the $\beta$ II and $\delta$ isoforms). It is assumed that activation of PKC interrupts insulin signaling by serine phosphorylation of insulin receptor substrate/IRS-1 resulting in a decrease in tyrosine phosphorylation of IRS-1. At the same time, activation of PKC also leads to production of inflammatory and proatherogenic proteins through activation of the IxB $\alpha / \mathrm{NF} x \mathrm{~B}$ pathway. $(1,2)$

Increasing evidence indicates that adipose tissue is an important source of cytokines and that adiposity contributes to development of proinflammatory milieu. Fat is both a dynamic endocrine organ as well a highly active metabolic tissue. Fat produces and secretes inflammatory factors, which are well known to play important roles in atherosclerotic process. Collectively, these factors are called adipocytokines or adipokines. These include TNF- $\alpha$, leptin, plasminogen activator inhibitor-1 (PAI-1), interleukin-6 (IL-6), resistin, and angiotensinogen. Adiponectin, a recently described adipokine of emerging importance, is distinct from the other known adipokines in that it alone appears to improve insulin sensitivity and inhibit vascular inflammation. Serum adiponectin levels are low in obese subjects but increase with weight loss. Adiponectin is an adipocyte-specific adipocytokine with anti-inflammatory, anti-diabetic and anti-atherogenic properties. Adiponectin and TNF- $\alpha$ inhibit each other's production in adipose tissue. Adiponectin expression is suppressed by IL-6 in adipose tissue. Adiponectin inhibits the TNF$\alpha$-induced monocyte attachment and endothelial adhesion molecule expression, macrophage-to-foam cell transformation, TNF- $\alpha$ expression in macrophages, and smooth muscle cell (SMC) proliferation $(3,4)$.
Adipocyte-specific fatty acid-binding protein (A-FABP) belongs to the fatty acid-binding protein super-family whose members have relative molecular masses (Mr) of $\sim 15000$, and it is highly expressed in adipose tissue. The fatty acid binding proteins aP2 (fatty acid binding protein [FABP]-4) and mal1 (FABP5) are closely related and both are expressed in adipocytes. A-FABP is a predominant cytosolic protein of mature adipocytes, accounting for $\sim 6 \%$ of total cellular proteins. This protein may be an important regulator of systemic insulin sensitivity and lipid and glucose metabolism. A-FABP is present in macrophages, which have striking similarities to adipocytes in biology and function. Accumulating evidence from animal experiments suggests that A-FABP is a central regulator of systemic insulin sensitivity, lipid metabolism, and inflammation, although the functional mechanisms remain poorly understood. Xu's study has demonstrated that A-FABP, traditionally thought to be a major cytoplasmic protein of adipocytes, is released from the cells and present in the blood stream. They also have found that A-FABP is one of the most abundant proteins secreted from adipocytes and confirmed the presence of circulating A-FABP in humans. They concluded that A-FABP is a circulating biomarker closely associated with obesity and components of the metabolic syndrome, and measurement of serum concentrations of A-FABP may be useful for the clinical diagnosis of obesity-related metabolic and cardiovascular disorders. $(5,6)$

Mal1 FABP5, also called keratinocyte lipid binding protein, is another member of the intracellular FABP family and has a high degree of homology to aP2. Whereas mal1 is expressed in differentiated adipocytes and macrophages; it is also found in other tissues and/or cell types, including skin, lens, lung, and brain. In wild type adipocytes, aP2 represents the majority of FABP, whereas mal1 constitutes a minor FABP fraction. Maeda's study demonstrated a significant role of mall in the pathogenesis of obesityrelated insulin resistance and type 2 diabetes. (6)

Visceral obesity is related to inflammation through a lot of complex factors which correlate each other. There is no study which measure correlation between lipolysis (reflected by FFA), lipid chaperone (reflected by FABP4 and FABP5), good adipokine (reflected by adiponectin) and inflammation (reflected by TNF- $\alpha$ and IL-6). Therefore, the aim of this study is to find correlations between FFA, FABP4, FABP5 and adiponectin, with TNF- $\alpha$ and IL- 6 among obese non diabetic males. 


\section{Methods}

We collected serum samples from 69 male subjects aged 30-60 years with non diabetic abdominal obesity. This study was an observational study with cross sectional design. Clinical examinations were conducted to select appropriate subjects. The criteria of obesity was waist circumference (WC) for Asian males $>90 \mathrm{~cm}$ (according to the International Diabetes Federation/ IDF criteria). The criteria of non diabetic condition was fasting plasma glucose $(\mathrm{FPG})<126 \mathrm{mg} / \mathrm{dL}$ (according to Perkumpulan Endokrinologi Indonesia/PERKENI criteria).

All of the study subjects were assessed after overnight fasting for at least 10 hours. The details of their anthropometric measurements (height, weight, BMI, waist circumference, and blood pressure) and measurements of routine biochemical variables (fasting glucose, fasting insulin, triglycerides, LDLand HDL-cholesterol, creatinine, serum glutamic oxaloacetic transaminase, and serum glutamic pyruvic transaminase) were done to all subjects. Insulin resistance was estimated with the homeostasis model assessment index (HOMA-IR), calculated as fasting glucose (in mmol/L) times fasting insulin (in $\mathrm{mIU} / \mathrm{L}$ ) divided by 22.5 .

FFA was measured by optimized enzymatic colorimetric assay for the determination of free fatty acids (= Non-Esterified Fatty Acids, NEFA) (Roche Diagnostics $\mathrm{GmbH}$, Germany). FABP4 was measured by Human FABP4 reagent with ELISA method (biotin labelled antibody based sandwich enzyme immunoassay for the quantitative measurement of human FABP4) (BioVendor Laboratory Medicine Inc, Czech Republic). FABP5 was measured by Human FABP5 reagent with ELISA (biotin labelled antibody based sandwich enzyme immunoassay for the quantitative measurement of human FABP5) (BioVendor Laboratory Medicine Inc, Czech Republic).

Adiponectin was measured based on the principle of a sandwich enzyme-linked immunosorbent assay (ELISA) (Sekisui Medical Co Ltd, Japan). Interleukin-6 Immunoassay is a solid phase ELISA designed to measure Interleukin- 6 which employs the quantitative sandwich enzyme immunoassay technique from $R \& D$ Systems Inc, USA. TNF- $\alpha$ Immunoassay is a solid phase ELISA designed to measure TNF- $\alpha$ which employs the quantitative sandwich enzyme immunoassay technique from R\&D Systems Inc, USA.

All statistical calculations were performed with the SPSS 11.5 statistical software package. We used the Pearson or Spearman's rho correlation coefficient to establish the association between various anthropometric and biochemical measures. We also used path analysis Lisrel 8.30 for Windows to strengthen the analysis.

\section{Results}

The details of anthropometric measurements (height, weight, BMI, waist circumference, and blood pressure); measurement of routine biochemical variables (fasting glucose, fasting insulin, triglycerides, LDLand HDL-cholesterol, creatinine, serum glutamic oxaloacetic transaminase, and serum glutamic pyruvic transaminase); and measurement of FFA, FABP4, FABP5, adiponectin, TNF- $\alpha$, and IL- 6 are all shown in Table 1 . We consider $\mathrm{p}<0.05$ as significant correlation.

This study revealed that there was no correlation between FFA, FABP4 and adiponectin with TNF- $\alpha$ and Interleukin-6, whereas there was weak but significant correlations between FABP 5 with TNF- $\alpha$ and Interleukin-6. This study also showed that there were weak but significant correlations between WC and hsCRP ( $\mathrm{r}=0.314, \mathrm{p}=0.000)$, WC and IL-6 ( $\mathrm{r}=$ $0.276, p=0.022)$, FFA and FABP4 $(r=0.263, p=0.029)$, FABP4 and WC $(r=0.249, \mathrm{p}=0.039), \mathrm{FABP} 4$ and BMI $(\mathrm{r}=0.311, \mathrm{p}=0.009)$, FABP5 and TNF- $\alpha(\mathrm{r}=0.408, \mathrm{p}=$ $0.000)$, FABP5 and FABP4 $(r=0.296, p=0.014)$, FABP5 and Interleukin- $6(\mathrm{r}=0.248, \mathrm{p}=0.04)$, Adiponectin and HDL-Cholesterol $(\mathrm{r}=0.301, \mathrm{p}=0.012)$.

From the correlation study, we suggest that there is correlation between abdominal obesity with inflammation. Inflammation processes are complex; and a lot of factors are involved and correlate each other.

All measurements were conducted in Prodia Research Laboratory, Jakarta, Indonesia. 


\section{Table 1. Results of Markers Biochemical Analysis}

\begin{tabular}{lc}
\hline PARAMETERS & MEAN \pm SD \\
\hline $\mathrm{n}=69$ & $38 \pm 6.2$ \\
\hline Age (years) & $98.9 \pm 6.5$ \\
\hline Waist Circumference $(\mathrm{cm})$ & $28.8 \pm 3.0$ \\
\hline Body Mass Index $(\mathrm{kg} / \mathrm{m} 2)$ & $115 \pm 7.6$ \\
\hline Systolic Blood Pressure $(\mathrm{mmHg})$ & $77 \pm 5.7$ \\
\hline Diastolic Blood Pressure $(\mathrm{mmHg})$ & $87.1 \pm 9.7$ \\
\hline Fasting Blood $\mathrm{Glucose}(\mathrm{mg} / \mathrm{dL})$ & $27.0 \pm 6.7$ \\
\hline SGOT $(\mathrm{U} / \mathrm{L})$ & $38.8 \pm 14.0$ \\
\hline SGPT $(\mathrm{U} / \mathrm{L})$ & $0.8 \pm 0.2$ \\
Bilirubin $(\mathrm{mg} / \mathrm{dL})$ & $1.0 \pm 0.2$ \\
\hline Creatinine $(\mathrm{mg} / \mathrm{dL})$ & $182.2 \pm 107.2$ \\
\hline Triglyceride $(\mathrm{mg} / \mathrm{dL})$ & $44.4 \pm 9.4$ \\
\hline HDL Cholesterol $(\mathrm{mg} / \mathrm{dL})$ & $10.4 \pm 6.4$ \\
\hline Insulin $(\mu / \mathrm{u} / \mathrm{mL})$ & $2.3 \pm 1.7$ \\
\hline HOMA IR & $3.6 \pm 1.4$ \\
\hline Adiponectin $(\mu \mathrm{g} / \mathrm{mL})$ & $0.4 \pm 0.1$ \\
\hline TNF- $\alpha(\mathrm{pg} / \mathrm{mL})$ & $21.8 \pm 8.7$ \\
\hline Interleukin-6 $(\mathrm{pg} / \mathrm{mL})$ & $2.0 \pm 0.8$ \\
\hline Free Fatty Acid $(\mathrm{mmol} / \mathrm{L})$ & \\
\hline FABP4 $(\mathrm{ng} / \mathrm{mL})$ & \\
\hline FABP5 $(\mathrm{ng} / \mathrm{mL})$ & \\
\hline
\end{tabular}

\section{Correlation Between FABP5 and Other Variables}

We used Pearson or Spearman correlation between FABP5 and other variables. According to the result, there are correlations between FABP5 and FABP4, IL- 6 and TNF- $\alpha$.

Table 2. Correlations Between FABP5 and Other Variables

\begin{tabular}{lcccc}
\hline & & FABP4 & TNF-a & Interleukin-6 \\
\hline FABP5 Correlation $(r)$ & & & \\
& $(p) 0$ & $.296^{*} 0$ & $.408^{* *} 0$ & $.248^{*}$ \\
& & .0000 & .040 \\
\hline${ }^{*} p<0.05$ & & & \\
${ }^{* *} p<0.001$ & & &
\end{tabular}




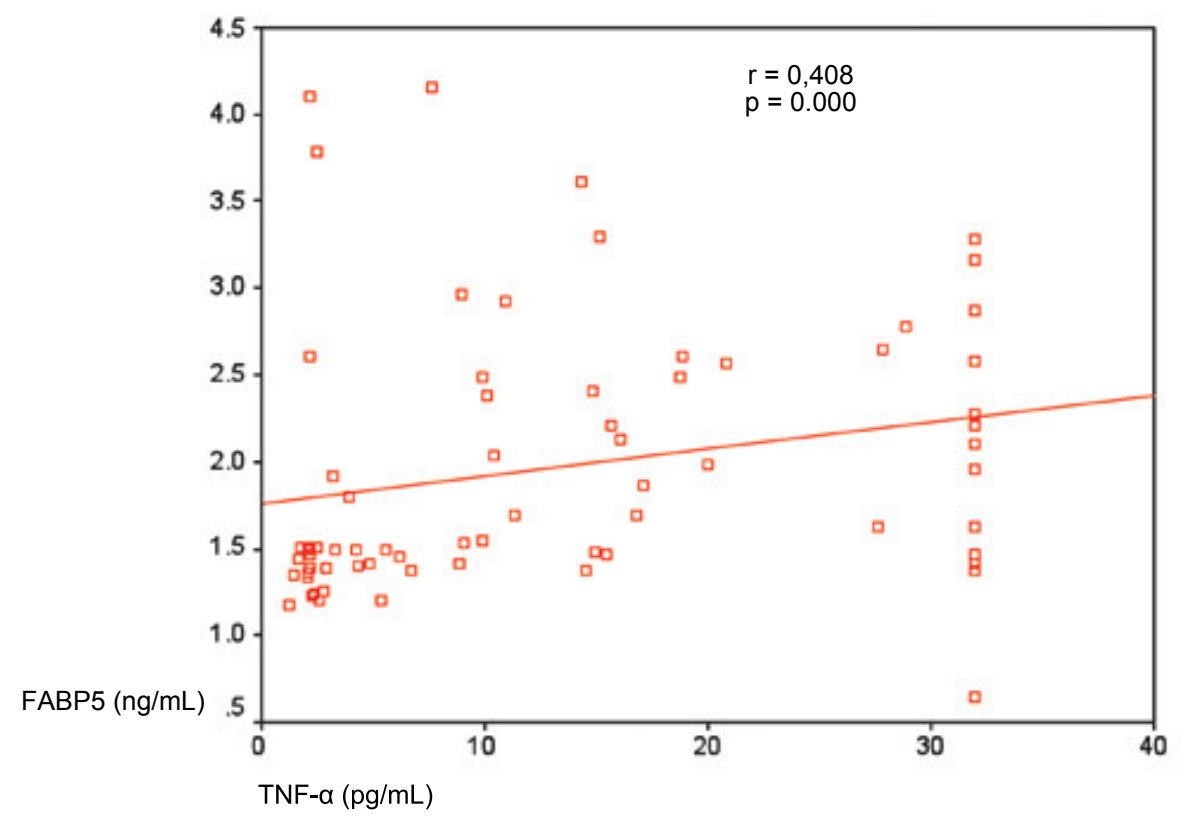

Figure 1. A Positive Correlation Between FABP5 and TNF- $\alpha$

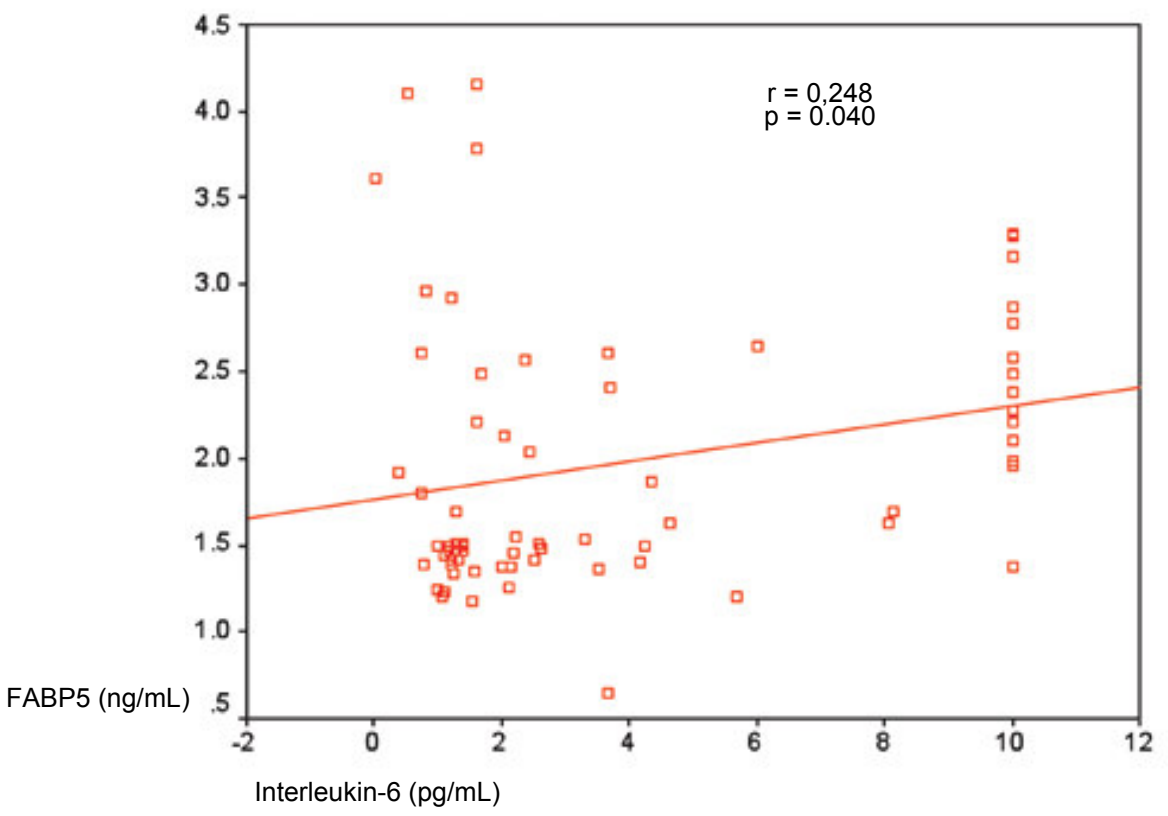

Figure 1. A Positive Correlation Between FABP5 and IL-6 


\section{Path Analysis using Lisrel 8.30 for Windows}

Our study showed a lot of factors having significant but weak correlations. We wanted to reveal the path between each factors to strengthen the analysis. Using Lisrel 8.30 for Windows, we showed path analysis between FFA, FABP4, FABP5, TNF- $\alpha$ and IL-6 (Figure 3).

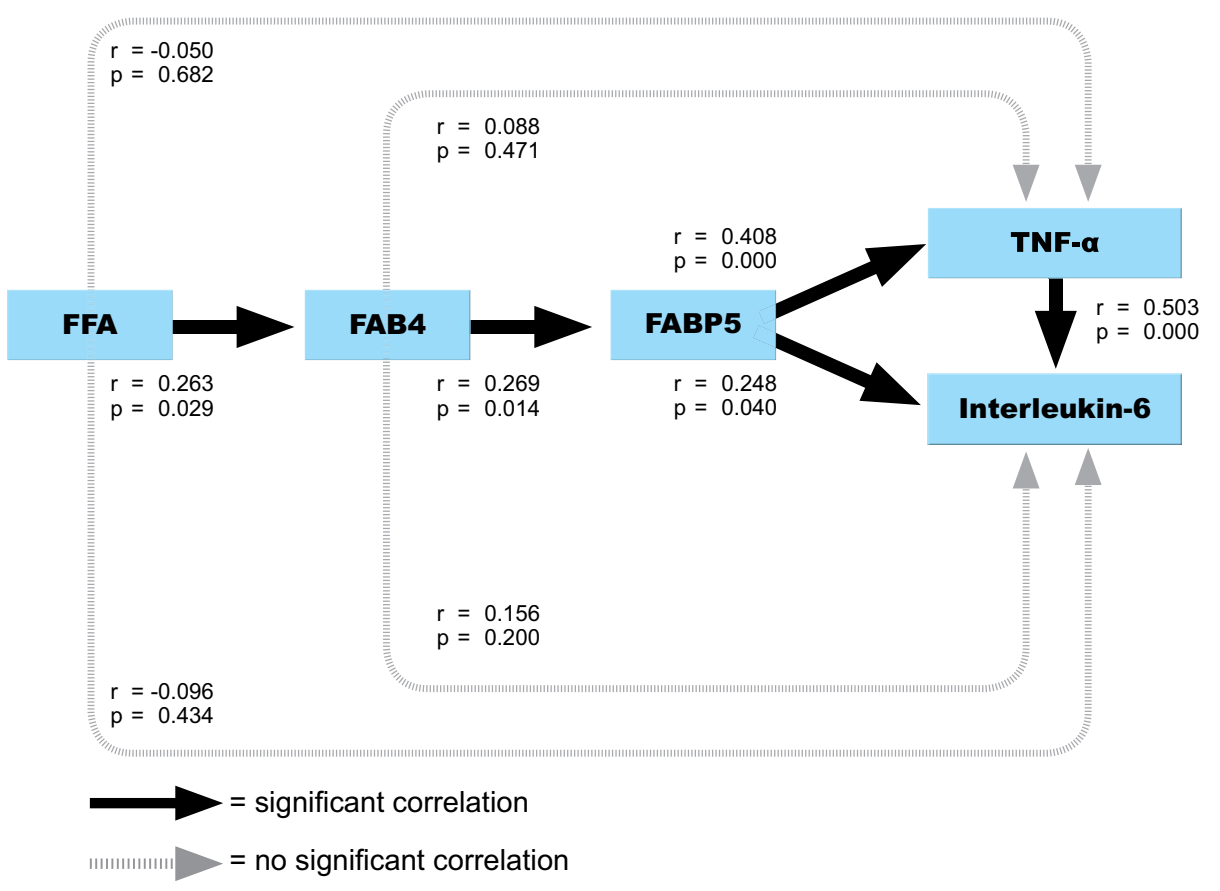

Figure 3. Path Analysis.

\section{Discussion}

In this study population, we found FFA serum concentration in obese nondiabetic males was $0.4 \pm 0.1$ $\mathrm{mmol} / \mathrm{L}$. According to Roden study, FFA concentration in obese nondiabetic individuals is $\sim 0.6-0.8 \mathrm{mmol} / \mathrm{L}$ while in overnight-fasted humans under physiological conditions, plasma FFA concentrations vary between 0.1 and $0.5 \mathrm{mmol} / \mathrm{L}$, primarily due to different rates of lipolysis. Our study of FFA serum concentration ranged physiological normal range. In adipocytes, glucose enhances the lipolysis rate, which may be attributed to increased expression of the rate-limiting enzyme for lipolysis, hormone-sensitive lipase. Our study population used nondiabetic individuals with normoglycemia $(87.1 \pm 9.7 \mathrm{mg} / \mathrm{dL})$, therefore we suggest that in our population study there is no increase in hormone sensitive lipase expression and no increase in lipolysis rate. We suggest that normal glucose concentration (no impaired fasting glucose) is related to normal lipolysis rate. Therefore we found FFA serum concentration to be still in physiological normal range in our study population . $(7,8)$
Our study showed that there was significant correlation between FFA and FABP4. The recent expansion of mechanistic insights into FABP action demonstrated that these lipid chaperones serve as upstream and critical modulators of many lipidsignaling cascades. FABPs are abundant cytoplasmic proteins that reversibly bind hydrophobic ligands such as saturated and unsaturated long chain fatty acids, plus eicosanoids such as hydroxyeicosatetraenoic acid, leukotrienes and prostaglandins. FABPs may actively facilitate the transport of lipids to specific compartments in the cell : such as to the mitochondria for oxidation; to the lipid droplet for storage; to enzymes for mediating activity; to the nucleus for lipid-mediated transcriptional regulation; or outside the cell to signal in an autocrine or paracrine fashion. (9).

In this study, no significant correlation was found between FFA-TNF- $\alpha$ and FFA-Interleukin-6. We suggest that FFA's role in inflammation may need another factor such as FABPs. 
We also found a correlation between FABP4BMI and FABP4-WC. According to Xu's study, the strong positive association between serum FABP4 concentrations and indicators of adiposity (BMI, waistto-hip ratio, $\mathrm{WC}$, and fat percentage) further suggests that adipose tissue, which is composed of adipocytes and macrophages, is probably the major contributor of FABP4 secreted into the circulation. (5)

In this study, FABP4 had significant correlation with FABP5, and there was correlation between FABP5 and TNF- $\alpha$ and FABP5 and Interleukin-6. FABP5, also called the keratinocyte lipid binding protein, mal1, is another member of the intracellular FABP family and has a high degree of homology to FABP4. These two proteins are $51 \%$ identical at the amino acid level and exhibit the same-barrel structure distinctive of all FABPs, suggestive of similar in vivo functions. Biochemically, FABP5 protein binds to the fatty acids that have been tested with similar selectivity and affinity as FABP4. Whereas FABP5 is expressed in differentiated adipocytes and macrophages, it is also found in other tissues and/or cell types, including skin, lens, lung, and brain. In wild-type adipocytes, FABP4 represents the majority of FABP, whereas FABP5 constitutes a minor FABP fraction. The ratio between FABP4 and FABP5 or absolute FABP abundance in the adipocyte and/or macrophages may be critical for the cellular physiology and responsible for the observed phenotypes. Fatty acid binding proteins FABP4 and FABP5 are highly similar in sequence and structure to each other and are coexpressed in adipocytes and macrophages. Although in adipocytes, FABP4 is the predominant FABP, FABP4-FABP5 stochiometry appears to be 1:1 in the macrophage under physiological conditions. The coordinated action of FABP4 and FABP5 are likely to play major roles in many components of the metabolic syndrome and define new pathways for prevention and/or treatment of these diseases. (10)

Because FABP4-FABP5 stochiometry appears to be $1: 1$ in the macrophage under physiological conditions, we suggest that expression of FABP5 is just like the same as FABP4. We suggest that in adipocytes, FABP5 has been shown to promote lipolysis, increase TNF- $\alpha$ expression, and contribute to insulin resistance. Macrophage FABP5 may influence foam cell formation and atherogenesis through effects on FFA shuttling that promote increased cholesteryl ester (CE) formation and/or effects on gene transcription that promote the production of inflammatory cytokines. Therefore in this study we found significant correlation between
FABP5-TNF- $\alpha$ and FABP5-Interleukin-6. (10)

We found no significant correlation between adiponectin-TNF- $\alpha$ and adiponectin-Interleukin-6. We suggest that this condition occured because inflammation in our study population had existed for a relatively short time (participants with age $<40$ years is $72.46 \%$ and age $>40$ years is $27.54 \%$ ) so the proinflammatory condition had nott yet induced the reduction of adiponectin concentration.

This study indicated that inflammation mechanism in human was complex and a lot of factors were involved and correlated each other. From clinical markers studied, we used Lisrel 8.30 for Windows to make path analysis. The analysis are shown in Figure 7.

We conclude that abdominal obesity might contribute to inflammation in obese nondiabetic males. Our study indicates that in abdominal obesity, FFA may induce inflammation through FABP4 and FABP5. Advancing our understanding of the function and measurement of FABP4 and FABP5 serum concentration will give insight into the clinical diagnosis of obesity-related metabolic disorders.

\section{Acknowledgement:}

We thank Endang Hoyaranda and The Prodia Foundation for Research and Training for their valuable help in supporting and conducting the many procedures of this research.

\section{References:}

1. Lenz A, Diamond FB. Obesity : The Hormonal Milieu. Curr Opin Endocrinol Diabetes Obes 2008; $15: 9-20$

2. Boden G. Free Fatty Acids as Target for Therapy. Curr Opin Endocrinol Diabetes 2004; 11:258-263

3. Lyon CJ, Law RE, Hsueh WA. Minireview: Adiposity, Inflammation, and Atherogenesis. Endocrinology 2003; 144(6) : 21952200

4. Ouchi N, Kihara S, Funahashi T, Matsuzawa Y, Walsh K. Obesity, Adiponectin and Vascular Inflammatory Disease. Curr Opin Lipidol 2003, 14 : 561-566

5. Xu A, Wang Y, Xu JY, et al. Adipocyte Fatty Acid-Binding Protein Is a Plasma Biomarker Closely Associated with Obesity and Metabolic Syndrome. Clin Chem 2006; 52(3) : 405-413

6. Maeda K, Uysal KT, Makowski L, et al. Role of the Fatty Acid Binding Protein mal1 in Obesity and Insulin Resistance. Diabetes 2003; $52: 300-307$

7. Roden M. How Free Fatty Acids Inhibit Glucose Utilization in Human Skeletal Muscle. News Physiol Sci 2004; 19 : 9296

8. Qvisth V, Hagstrom-Toft E, Enoksson S, et al. Combined Hyperinsulinemia and Hyperglycemia, But Not Hyperinsulinemia Alone, Suppress Human Skeletal Muscle Lipolytic Activity In Vivo. J Clin Endocrinol Metab 2004; 89(9) : 4693-4700

9. Makowski L, Hotamisligil GS. The Role of Fatty Acid Binding Proteins in Metabolic Syndrome and Atherosclerosis. Curr Opin Lipidol 2006; 16 : 543-548

10. Boord JB, Fazio S, Linton MF. Cytoplasmic Fatty Acid-Binding Protein : Emerging Roles in Metabolism and Atherosclerosis. Curr Opin Lipidol, 2002; 13 : 141-147 